Document downloaded from:

http://hdl.handle.net/10251/64688

This paper must be cited as:

Artidiello Moreno, SDJ.; Cordero Barbero, A.; Torregrosa Sánchez, JR.; Vassileva, MP. (2015). Multidimensional generalization of iterative methods for solving nonlinear problems by means of weight-function procedure. Applied Mathematics and Computation. 268:10641071. doi:10.1016/j.amc.2015.07.024.

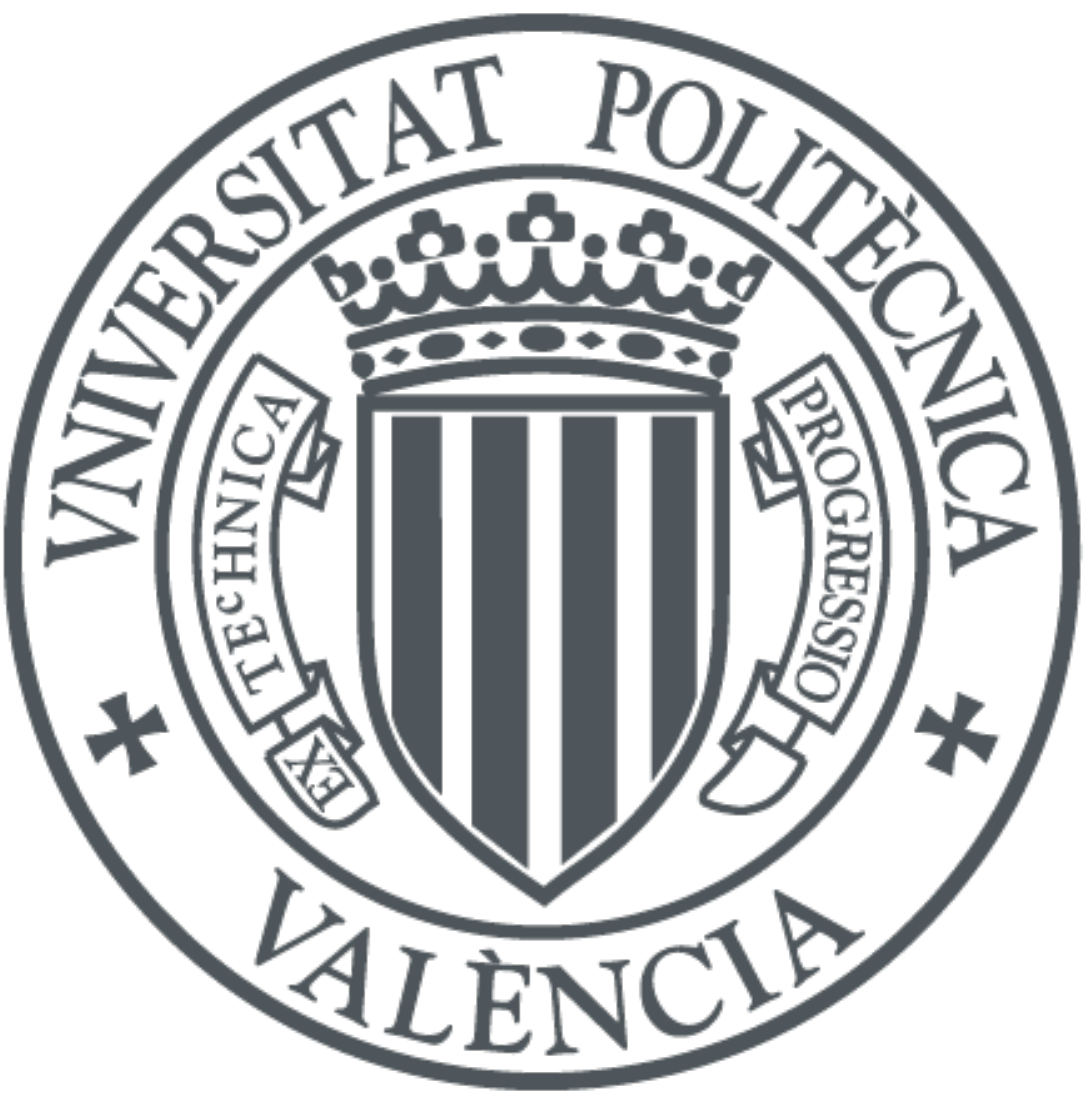

The final publication is available at

https://dx.doi.org/10.1016/j.amc.2015.07.024

Copyright Elsevier

Additional Information 


\title{
Multidimensional generalization of iterative methods for solving nonlinear problems by means of weight-function procedure
}

\author{
S. Artidiello ${ }^{\mathrm{a}}$, Alicia Cordero ${ }^{\mathrm{b}}$, Juan R. Torregrosa ${ }^{\mathrm{b}, *}$, Maria P. Vassileva $^{\mathrm{a}}$ \\ ${ }^{a}$ Instituto Tecnologico de Santo Domingo (INTEC), Santo Domingo, República Dominicana \\ ${ }^{b}$ Instituto Universitario de Matemática Multidisciplinar, Universitat Politècnica de València, València, Spain
}

\begin{abstract}
In this paper, from Traub's method and by applying weight function technique, a bi-parametric family of predictor-corrector iterative schemes with optimal fourth-order of convergence, for solving nonlinear equations, is presented. By using some algebraic manipulations and a divided difference operator, we extend this family to the multidimensional case, preserving its order of convergence. Some numerical test are made in order to confirm the theoretical results and to compare the new methods with other known ones.
\end{abstract}

Keywords: Nonlinear system, optimal order, weight function procedure, divided difference operator, efficiency index

AMS Subject Classification: 65H05, 65H10.

\section{Introduction}

Solving nonlinear equations and systems is an important task in theory and practice for many branches of Science and Engineering. Sometimes the nonlinear system is approximated by a system of linear equations but, when this approximation is not satisfactory, the problem must be confronted directly.

A way to design iterative methods for solving nonlinear systems is to adapt the methods of the scalar case, but not always these schemes can be extended to multidimensional case, at least, in a direct form. There exist some methods in the scalar case which are directly extended to system of nonlinear equations, others have been specially designed for systems. In the design of iterative methods we must bear in mind that, while in the scalar case functional evaluation of the nonlinear function and its derivatives have the same computational cost, this is not true in the vectorial case. For solving nonlinear systems, it is much more efficient a scheme that needs an evaluation of the Jacobian matrix and two functions, per iteration, than a scheme with two evaluations of the Jacobian matrix and an evaluation of the function per iteration; being both them, in the scalar case, optimal in the sense of Kung-Traub conjecture [1]. They conjectured that an iterative method that needs $d$ functional evaluations per iteration has, at most, order $2^{d-1}$; when this bound is reached, the method is called optimal. Moreover, recently many researchers are working on the extension to systems of one-dimensional schemes which, a priori, do not have direct extension to multidimensional case (see, for example, [2, 3, 4]).

In the last years, the weight function procedure has been used in the development of high order iterative methods for systems, see for example the papers of Sharma et al. [5], [6], the schemes published in [7], where

\footnotetext{
This research was partially supported by Ministerio de Economía y Competitividad MTM2014-52016-C02-02 and FONDOCYT 2014-1C1-088, República Dominicana.

${ }^{*}$ Corresponding author

Email addresses: santiago.artidiello@intec.edu.do (S. Artidiello), acordero@mat.upv.es (Alicia Cordero), jrtorre@mat.upv.es (Juan R. Torregrosa), maria.penkova@intec.edu.do (Maria P. Vassileva)
} 
the authors apply them for solving the problem of preliminary orbit determination of artificial satellites, and the work of Abad et al. [8], where the authors apply the designed methods on the Global Positioning System.

The proliferation of iterative methods for solving nonlinear equations has been spectacular in the last years (in [9] we can see a good review). Some of these methods can be transferred easily to the context of nonlinear systems, but others, at least apparently, cannot be extended to multidimensional case. In this paper, we want to prove that this translation is possible, at least for many of the known multipoint iterative methods designed for nonlinear equations, making use of tools such as the divided difference operator. We understand that this translation only has interest if the order of convergence is preserved, so we will pay attention to the conditions under which the order is kept.

The rest of the paper is organized as follows: in Section 2 we design a fourth-order parametric family for solving nonlinear equation and we analyze its order of convergence. In Section 3 the previous family is generalized for solving nonlinear systems preserving its order of convergence. Section 4 is devoted to the numerical tests that confirm the theoretical results and allow us to compare the proposed schemes with some known ones. The paper finishes with some conclusions and remarks.

\section{Design of a scalar parametric family}

We are going to design a parametric family of iterative methods for finding a simple root $\xi$ of a nonlinear equation $f(x)=0$, where $f$ is a real function $f: I \subseteq \mathbb{R} \longrightarrow \mathbb{R}$, defined in an open interval $I$.

We consider the multipoint expression:

$$
\begin{aligned}
y_{k} & =x_{k}-\frac{f\left(x_{k}\right)}{f^{\prime}\left(x_{k}\right)}, \\
x_{k+1} & =y_{k}-\frac{f\left(y_{k}\right)}{f^{\prime}\left(x_{k}\right)},
\end{aligned}
$$

corresponding to the well known Traub's method (see [10]), with order of convergence three. This scheme is not optimal in the sense of Kung-Traub conjecture. By using a weight function, we propose the following generalization of (1):

$$
\begin{aligned}
y_{k} & =x_{k}-\alpha \frac{f\left(x_{k}\right)}{f^{\prime}\left(x_{k}\right)}, \\
x_{k+1} & =y_{k}-h\left(\mu\left(x_{k}\right)\right) \frac{f\left(y_{k}\right)}{f^{\prime}\left(x_{k}\right)},
\end{aligned}
$$

where $h\left(\mu\left(x_{k}\right)\right)$ is a function of real variable $\mu\left(x_{k}\right)=\frac{a_{1} f\left(x_{k}\right)+a_{2} f\left(y_{k}\right)}{b_{1} f\left(x_{k}\right)+b_{2} f\left(y_{k}\right)}$ and $\alpha, a_{1}, a_{2}, b_{1}$ and $b_{2}$ are real parameters. In the following result, we present the conditions that function $h$ must satisfy for obtaining a class of iterative methods of order of convergence at least four, becoming optimal schemes.

Theorem 1. Let $f: I \subseteq \mathbb{R} \rightarrow \mathbb{R}$ be a sufficiently differentiable function in an open interval $I$, such that $\xi \in I$ is a simple solution of the nonlinear equation $f(x)=0$. Let $h: \mathbb{R} \rightarrow \mathbb{R}$ be any sufficiently differentiable function such that $h(c)=1, h^{\prime}(c)=\frac{2 b_{1}^{2}}{a_{2} b_{1}-a_{1} b_{2}},\left|h^{\prime \prime}(c)\right|<\infty$, where $c=\frac{a_{1}}{b_{1}}$. If $x_{0}$ is close enough to $\xi$, $\alpha=1$ and $b_{1} \neq 0$ then method defined by (2) has fourth-order of convergence and its error equation is:

$$
e_{k+1}=\frac{1}{2 b_{1}^{4}}\left[\left(10 b_{1}^{4}+4 b_{1}^{3} b_{2}+h^{\prime \prime}(c)\left(-a_{2}^{2} b_{1}^{2}+2 a_{1} a_{2} b_{1} b_{2}-a_{1}^{2} b_{2}^{2}\right)\right) c_{2}^{3}-2 b_{1}^{4} c_{2} c_{3}\right] e_{k}^{4}+\mathcal{O}\left(e_{k}^{5}\right)
$$


where $c_{k}=\frac{1}{k !} \frac{f^{(k)}(\xi)}{f^{\prime}(\xi)}, k=2,3, \ldots$ and $e_{k}=x_{k}-\xi$.

Proof. To prove the local order of convergence we use the Taylor series expansion of the functions involved around the iterative expression

$$
\begin{aligned}
& f\left(x_{k}\right)=f^{\prime}(\xi)\left(e_{k}+c_{2} e_{k}^{2}+c_{3} e_{k}^{3}+c_{4} c_{k}^{4}\right)+\mathcal{O}\left(e_{k}^{5}\right), \\
& f^{\prime}\left(x_{k}\right)=f^{\prime}(\xi)\left(1+2 c_{2} e_{k}+3 c_{3} e_{k}^{2}+4 c_{4} e_{k}^{3}\right)+\mathcal{O}\left(e_{k}^{4}\right) .
\end{aligned}
$$

By substituting these expressions in the first step of (2) we obtain

$$
y_{k}=\xi-(1-\alpha) e_{k}+\alpha c_{2} e_{k}^{2}-2 \alpha\left(c_{2}^{3}-c_{3}\right) e_{k}^{3}+\alpha\left(-4 c_{2}^{3}+7 c_{2} c_{3}-3 c_{4}\right) e_{k}^{4}+\mathcal{O}\left(e_{k}^{5}\right) .
$$

So,

$$
f\left(y_{k}\right)=f^{\prime}(\xi)\left(A_{1} e_{k}+A_{2} e_{k}^{2}+A_{3} e_{k}^{3}+A_{4} e_{k}^{4}\right)+\mathcal{O}\left(e_{k}^{5}\right),
$$

where $A_{1}=1-\alpha, A_{2}=\left(1-\alpha+\alpha^{2}\right) c_{2}, A_{3}=-2 \alpha^{2} c_{2}^{2}+\left(1-\alpha+3 \alpha^{2}-\alpha^{3}\right) c_{3}$ and $A_{4}=5 \alpha^{2} c_{2}^{3}-$ $\alpha^{2}(10-3 \alpha) c_{2} c_{3}+\left(1-\alpha+6 \alpha^{2}-4 \alpha^{3}+\alpha^{4}\right) c_{4}$. Hence, using the expansions of $f\left(x_{k}\right)$ and $f\left(y_{k}\right)$, we obtain the expression of variable $\mu\left(x_{k}\right)$ :

$$
\mu\left(x_{k}\right)=\frac{a_{1} f\left(x_{k}\right)+a_{2} f\left(y_{k}\right)}{b 1 f\left(x_{k}\right)+b_{2} f\left(y_{k}\right)}=B_{0}+B_{1} e_{k}+B_{2} e_{k}^{2}+B_{3} e_{k}^{3}+\mathcal{O}\left(e_{k}^{4}\right)
$$

where $B_{0}=\frac{m_{1}}{m_{2}}$, with $m_{1}=a_{1}+a_{2}-\alpha a_{2}$ and $m_{2}=b_{1}+b_{2}-\alpha b_{2}, B_{1}=\frac{\alpha^{2} m_{3}}{m_{2}^{3}} c_{2}$, with $m_{3}=a_{2} b_{1}-a_{1} b_{2}$, $B_{2}=\frac{\alpha^{2} m_{3}}{m_{2}^{3}}\left[\left(3 b_{1}+\left(3-3 \alpha+\alpha^{2}\right) c_{2}^{2}+(\alpha-3) m_{2} c_{3}\right]\right.$ and $B_{3}=\frac{\alpha^{2} m_{3}}{m_{2}^{4}}\left(n_{1} c_{2}^{3}+n_{2} c_{2} c_{3}+2 n_{3} c_{2}\right)$ with $n_{1}=8 b_{1}^{2}+2\left(3-3 \alpha+\alpha^{2}\right) b_{2}, n_{2}=(2 \alpha-7) b_{1}^{2}+\left(\alpha^{3}-7 \alpha^{2}+18 \alpha-14\right) b_{1} b_{2}-\left(\alpha^{4}-6 \alpha^{3}+14 \alpha^{2}-16 \alpha+7\right) b_{2}^{2}$ and $n_{3}=m_{2}^{2}\left(6-4 \alpha+\alpha^{2}\right)$.

Taking into account that $\mu\left(x_{k}\right) \rightarrow c=\frac{a_{1}}{b_{1}}$, when $x_{k} \rightarrow \xi$, and by using Taylor series expansion of $h\left(\mu\left(x_{k}\right)\right)$ around $c$, we obtain

$$
h\left(\mu\left(x_{k}\right)\right) \approx h(c)+h^{\prime}(c)\left(\mu\left(x_{k}\right)-c\right)+\frac{h^{\prime \prime}(c)}{2}\left(\mu\left(x_{k}\right)-c\right)^{2}
$$

and we obtain the error equation of (2) in the form:

$$
e_{k+1}=(1-\alpha) D_{1} e_{k}+D_{2} e_{k}^{2}+D_{3} e_{k}^{3}+D_{4} e_{k}^{4}+\mathcal{O}\left(e_{k}^{5}\right)
$$

We observe that if $\alpha=1$ the order of convergence is at least 2. Then, with this value of $\alpha$, the error equation takes the form $e_{k+1}=D_{2}^{\prime} e_{k}^{2}+D_{3}^{\prime} e_{k}^{3}+D_{4}^{\prime} e_{k}^{4}+\mathcal{O}\left(e_{k}^{5}\right)$, where $D_{2}^{\prime}=(1-h(c)) c_{2}$. So, if $h(c)=1$ we will obtain order of convergence at least 3 and the error equation can be expressed as $e_{k+1}=D_{3}^{\prime \prime} e_{k}^{3}+D_{4}^{\prime \prime} e_{k}^{4}+\mathcal{O}\left(e_{k}^{5}\right)$, where $D_{3}^{\prime \prime}=\frac{1}{b_{1}^{2}}\left(2 b_{1}^{2}-m_{3} h^{\prime}(c)\right) c_{2}^{2}$. Now, if we take $h^{\prime}(c)=2 b_{1}^{2} / m_{3}$ we obtain an order of convergence at least 4 and the error equation takes the form (3).

We note that the family of iterative methods published by the authors in [11] is a particular case of (2) with $a_{1}=0$. The class of schemes presented by Chun in [12] is also a particular case of (2) with $a_{1}=b_{2}=0$. On the other hand, if $a_{1}=0, a_{2}=1, b_{1}=1$ and we take $h(\mu)=1+2 \mu$, King's family [13] is obtained.

For the numerical tests we will use the following members of family (2): 
- if $a_{1}=0, a_{2}=2, b_{1}=1 / 2, b_{2}=0$ and $h(\mu)=\frac{6}{6-3 \mu-\mu^{2}}$, then the iterative scheme has the expression

$$
x_{k+1}=y_{k}-\frac{2 f\left(x_{k}\right)+3 f\left(y_{k}\right)}{2 f\left(x_{k}\right)-f\left(y_{k}\right)} \frac{f\left(y_{k}\right)}{f^{\prime}\left(x_{k}\right)},
$$

- if $a_{1}=1, a_{2}=0, b_{1}=1, b_{2}=-1$ and $h(\mu)=-1+2 \mu$, then the iterative method takes the form

$$
x_{k+1}=y_{k}-\frac{f\left(x_{k}\right)+f\left(y_{k}\right)}{f\left(x_{k}\right)-f\left(y_{k}\right)} \frac{f\left(y_{k}\right)}{f^{\prime}\left(x_{k}\right)},
$$

where $y_{k}$ denotes in both cases Newton's iteration. These iterative schemes are denoted by ME1 and ME2, respectively.

\section{Generalization to multidimensional case}

In this section we aim to give a generalization of the family of methods (2) obtained in the previous section, for solving a nonlinear system $F(x)=0$, where $F: D \subseteq \mathbb{R}^{n} \rightarrow \mathbb{R}^{n}$, preserving the order convergence. The idea that we will present can be applied, with the same objective, on many iterative methods designed for solving nonlinear equations.

To extend the iterative scheme (2), with $\alpha=1$, to the multidimensional case, it is necessary to rewrite the iterative expression so that no functional evaluation of the nonlinear function remains in the denominator (as the authors did in [2,3]). To achieve this objective, consider the first step of the iterative process $y_{k}=x_{k}-\frac{f\left(x_{k}\right)}{f^{\prime}\left(x_{k}\right)}$ that can be rewritten as $f\left(x_{k}\right)=\left(x_{k}-y_{k}\right) f^{\prime}\left(x_{k}\right)$. Using this, we can rewrite the quotient $\frac{f\left(y_{k}\right)}{f\left(x_{k}\right)}$ as

$$
\frac{f\left(y_{k}\right)}{f\left(x_{k}\right)}=\frac{f\left(x_{k}\right)+f\left(y_{k}\right)-f\left(x_{k}\right)}{f\left(x_{k}\right)}=1-\frac{f\left[x_{k}, y_{k}\right]}{f^{\prime}\left(x_{k}\right)} .
$$

So, variable $\mu$ can be written as

$$
\mu\left(x_{k}\right)=\frac{a_{1}+a_{2} \frac{f\left(y_{k}\right)}{f\left(x_{k}\right)}}{b_{1}+b_{2} \frac{f\left(y_{k}\right)}{f\left(x_{k}\right)}}=\frac{\left(a_{1}+a_{2}\right) f^{\prime}\left(x_{k}\right)-a_{2} f\left[x_{k}, y_{k}\right]}{\left(b_{1}+b_{2}\right) f^{\prime}\left(x_{k}\right)-b_{2} f\left[x_{k}, y_{k}\right]}
$$

By using this transformation, the proposed family (2), with $\alpha=1$, is generalizable to several variables in the following way:

$$
\begin{aligned}
y^{(k)} & =x^{(k)}-\left[F^{\prime}\left(x^{(k)}\right)\right]^{-1} F\left(x^{(k)}\right), \\
x^{(k+1)} & =y^{(k)}-H\left(\eta^{(k)}\right)\left[F^{\prime}\left(x^{(k)}\right)\right]^{-1} F\left(y^{(k)}\right),
\end{aligned}
$$

where $\eta^{(k)}=M^{-1} N, M=\left(b_{1}+b_{2}\right) I-b_{2} T, N=\left(a_{1}+a_{2}\right) I-a_{2} T, T=\left[F^{\prime}\left(x^{(k)}\right)\right]^{-1}\left[x^{(k)}, y^{(k)} ; F\right]$ and $a_{1}, a_{2}, b_{1}$ and $b_{2}$ are real parameters.

Let us denote by $F^{\prime}\left(x^{(k)}\right)$ the Jacobian matrix of $F$ evaluated in the $k$ th-iteration and by $\left[x^{(k)}, y^{(k)} ; F\right]$ the divided difference operator defined in [14] as the function $[\cdot, \cdot ; F]: D \times D \subset \mathbb{R}^{n} \times \mathbb{R}^{n} \rightarrow \mathcal{L}\left(\mathbb{R}^{n}\right)$ that satisfies $[x, y ; F](x-y)=F(x)-F(y), \forall x, y \in D$. 
Since the analysis of the local convergence is based on the Taylor series expansion around the solution, we need to obtain the corresponding development of the divided difference operator. To achieve this, we use the Genocchi-Hermite formula (see [15])

$$
[x, x+h ; F]=\int_{0}^{1} F^{\prime}(x+t h) d t
$$

and, by developing $F^{\prime}(x+t h)$ in Taylor series around $x$, we obtain

$$
\int_{0}^{1} F^{\prime}(x+y h) d t=F^{\prime}(x)+\frac{1}{2} F^{\prime \prime}(x) h+\frac{1}{6} F^{\prime \prime \prime}(x) h^{2}+\mathcal{O}\left(h^{3}\right) .
$$

Defining $e=x-\xi$ and assuming that $F^{\prime}(\xi)$ is nonsingular, we have:

$$
\begin{aligned}
& F(x)=F^{\prime}(\xi)\left(e+C_{2} e^{2}+C_{3} e^{3}+C_{4} e^{4}\right)+\mathcal{O}\left(e^{5}\right), \\
& F^{\prime}(x)=F^{\prime}(\xi)\left(I+2 C_{2} e+3 C_{3} e^{2}+4 C_{4} e^{3}\right)+\mathcal{O}\left(e^{4}\right), \\
& F^{\prime \prime}(x)=F^{\prime}(\xi)\left(2 C_{2}+6 C_{3} e+12 C_{4} e^{2}\right)+\mathcal{O}\left(e^{3}\right), \\
& F^{\prime \prime \prime}(x)=F^{\prime}(\xi)\left(6 C_{3}+24 C_{4} e\right)+\mathcal{O}\left(e^{2}\right),
\end{aligned}
$$

where $I$ denotes the identity matrix of size $n \times n$ and $C_{q}=\frac{1}{q !}\left[F^{\prime}(\xi)\right]^{-1} F^{(q)}(\xi), q \geq 2$. Replacing these developments in the formula of Genocchi-Hermite and denoting the second point of the divided difference by $y=x+h$ and the error of the first step by $e_{y}=y-\xi$, we have

$$
[x, y ; F]=F^{\prime}(\xi)\left[I+C_{2}\left(e_{y}+e\right)+C_{3} e^{2}\right]+O\left(e^{3}\right) .
$$

In particular, if $y$ is the approximation of the solution provided by Newton's method, i.e., $h=x-y=$ $\left[F^{\prime}(x)\right]^{-1} F(x)$, we obtain

$$
[x, y ; F]=F^{\prime}(\xi)\left[I+C_{2} e+\left(C_{2}^{2}+C_{3}\right) e^{2}\right]+O\left(e^{3}\right) .
$$

On the other hand, if we denote by $X=\mathbb{R}^{n \times n}$ the space of all $n \times n$ real matrices, the weight function in this context is $H: X \rightarrow X$ such that

(i) $H^{\prime}(u)(v)=H_{1} u v$, being $H^{\prime}$ the first derivative of $H, H^{\prime}: X \rightarrow \mathcal{L}(X), H_{1} \in \mathbb{R}$ and $\mathcal{L}(X)$ denotes the space of linear mappings from $X$ to itself.

(ii) $H^{\prime \prime}(u, v)(w)=H_{2} u v w$, being $H^{\prime \prime}$ the second derivative of $H, H^{\prime \prime}: X \times X \rightarrow \mathcal{L}(X)$ and $H_{2} \in \mathbb{R}$.

Then, the Taylor expansion of $H$ around the identity matrix gives

$$
H\left(\eta^{(k)}\right) \approx H(I)+H_{1}\left(\eta^{(k)}-I\right)+\frac{1}{2} H_{2}\left(\eta^{(k)}-I\right)^{2} .
$$

In order to establish the order of convergence of (4), we present the following result in whose proof we use the tools introduced in [16].

Theorem 2. Let $F: D \subseteq \mathbb{R}^{n} \rightarrow \mathbb{R}^{n}$ be a sufficiently differentiable function in a convex set $D$ and $\xi \in D$ a solution of $F(x)=0$. Let us suppose that $F^{\prime}(x)$ is continuous and nonsingular in $\xi$ and $x^{(0)}$ is close enough to $\xi$. Then, the sequence $\left\{x^{(k)}\right\}_{k \geq 0}$ obtained from (4) converge to $\xi$, with order of convergence four, if matrix 
function $H$ satisfies $H(c)=I, H^{\prime}(c)=H_{1} I$ and $\left\|H^{\prime \prime}(c)\right\|<\infty$, where $c=\frac{a_{1}}{b_{1}} I, H_{1}=\frac{2 b_{1}^{2}}{a_{2} b_{1}-a_{1} b_{2}}$ and $b_{1} \neq 0$. Furthermore, the error equation is

$$
e^{(k+1)}=\frac{1}{2 b_{1}^{4}}\left[\left(10 b_{1}^{4}+4 b_{1}^{3} b_{2}-H^{\prime \prime}(c)\left(a_{2}^{2} b_{1}^{2}-2 a_{1} a_{2} b_{1} b_{2}+a_{1}^{2} b_{2}^{2}\right)\right) C_{2}^{3}-2 b_{1}^{4} C_{2} C_{3}\right] e^{(k)^{4}}+\mathcal{O}\left(e^{(k)^{5}}\right),
$$

where $\left.C_{j}=\frac{1}{j !}\left[F^{\prime}(\xi)\right]^{-1}\right] F^{(j)}(\xi), j=2,3, \ldots$ and $e^{(k)}=x^{(k)}-\xi$.

Proof. Using the Taylor series expansion of $F\left(x^{(k)}\right)$ and $F^{\prime}\left(x^{(k)}\right)$ around $\xi$, we obtain

$$
\begin{aligned}
& F\left(x^{(k)}\right)=F^{\prime}(\xi)\left(e^{(k)}+C_{2} e^{(k)^{2}}+C_{3} e^{(k)^{3}}+C_{4} e^{(k)^{4}}+C_{5} e^{(k)^{5}}\right)+\mathcal{O}\left(e^{(k)^{6}}\right), \\
& F^{\prime}\left(x^{(k)}\right)=F^{\prime}(\xi)\left(I+2 C_{2} e^{(k)}+3 C_{3} e^{(k)^{2}}+4 C_{4} e^{(k)^{3}}+5 C_{5} e^{(k)^{4}}\right)+\mathcal{O}\left(e^{(k)^{4}}\right), \\
& {\left[F^{\prime}\left(x^{(k)}\right)\right]^{-1}=\left(I+X_{2} e^{(k)}+X_{3} e^{(k)^{2}}+X_{4} e^{(k)^{3}}\right)\left[F^{\prime}(\xi)\right]^{-1}+\mathcal{O}\left(e^{(k)^{4}}\right),}
\end{aligned}
$$

where $X_{2}=-2 C_{2}, X_{3}=4 C_{2}^{2}-3 C_{3}$ and $X_{4}=-8 C_{2}^{3}+6 C_{2} C_{3}+6 C_{3} C_{2}-4 C_{4}$ are obtained requiring $\left[F^{\prime}\left(x^{(k)}\right)\right]^{-1} F\left(x^{(k)}\right)=I$. Substituting these Taylor expansions in the first step of (4), we get

$$
y^{(k)}=\xi+A_{2} e^{(k)^{2}}+A_{3} e^{(k)^{3}}+A_{4} e^{(k)^{4}}+O\left(e^{(k)^{5}}\right),
$$

where $A_{2}=C_{2}, A_{3}=2\left(C_{3}-C_{2}^{2}\right)$ and $A_{4}=4 C_{2}^{3}-4 C_{2} C_{3}-3 C_{3} C_{2}+3 C_{4}$.

Using again Taylor series for developing $F\left(y^{(k)}\right)$ around $\xi$, we have

$$
F\left(y^{(k)}\right)=F^{\prime}(\xi)\left[A_{2} e^{(k)^{2}}+A_{3} e^{(k)^{3}}+A_{5} e^{(k)^{4}}\right]+O\left(e^{(k)^{5}}\right),
$$

where $A_{5}=5 C_{2}^{3}-4 C_{2} C_{3}-3 C_{3} C_{2}+3 C_{4}$.

Now, by using expressions (5) and (6), we obtain:

$$
\begin{aligned}
{\left[x^{(k)}, y^{(k)} ; F\right] } & =F^{\prime}(x)+\frac{1}{2} F^{\prime \prime}(x) h+\frac{1}{6} F^{\prime \prime \prime}(x) h^{2}+\mathcal{O}\left(h^{3}\right) \\
& =F^{\prime}(\xi)\left(I+P_{1} e^{(k)}+P_{2} e^{(k)^{2}}+P_{3} e^{(k)^{3}}\right)+\mathcal{O}\left(e^{(k)^{4}}\right),
\end{aligned}
$$

where $h=y^{(k)}-x^{(k)}=-\left[F^{\prime}\left(x^{(k)}\right)\right]^{-1} F\left(x^{(k)}\right), P_{1}=C_{2}, P_{2}=C_{2}^{2}+C_{3}$ and $P_{3}=-2 C_{2}^{3}+2 C_{2} C_{3}+$ $C_{3} C_{2}+C_{4}$. For obtaining the expressions of $M$ and $N$, we need the Taylor expansion of $T$

$$
T=\left[F^{\prime}\left(x^{(k)}\right)\right]^{-1}\left[x^{(k)}, y^{(k)} ; F\right]=I+T_{1} e^{(k)}+T_{2} e^{(k)^{2}}+T_{3} e^{(k)^{3}}+\mathcal{O}\left(e^{(k)^{4}}\right),
$$

being $T_{1}=P_{1}+X_{2}$ and $T_{m}=P_{m}+\sum_{j=1}^{m} X_{j} P_{m-j+1}+X_{m+1}, m=2,3, \ldots$ Using this result, we obtain the expressions for $M$ and $N$ :

$$
\begin{aligned}
& M=\left(b_{1}+b_{2}\right) I-b_{2} T=b_{1} I+M_{1} e^{(k)}+M_{2} e^{(k)^{2}}+M_{3} e^{(k)^{3}}+M_{4} e^{(k)^{4}}+\mathcal{O}\left(e^{(k)^{5}}\right), \\
& N=\left(a_{1}+a_{2}\right) I-a_{2} T=a_{1} I+N_{1} e^{(k)}+N_{2} e^{(k)^{2}}+N_{3} e^{(k)^{3}}+N_{4} e^{(k)^{4}}+\mathcal{O}\left(e^{(k)^{5}}\right),
\end{aligned}
$$

where $M_{j}=-b_{2} T_{j}, N_{j}=-a_{2} T_{j}, j=1,2, \ldots$. Let us consider the inverse of operator $M$

$$
M^{-1}=Y_{1}+Y_{2} e^{(k)}+Y_{3} e^{(k)^{2}}+Y_{4} e^{(k)^{3}}+Y_{5} e^{(k)^{4}}+\mathcal{O}\left(e^{(k)^{5}}\right) .
$$


For determining coefficients $Y_{j}$ we use condition $M^{-1} M=I$. Then, $Y_{1}=\frac{1}{b_{1}} I$ and $Y_{s}=-\frac{1}{b_{1}} \sum_{j=1}^{s} Y_{j} M_{s-j}$, $s=2,3, \ldots$

Finally, we express variable $\eta\left(x^{(k)}\right)$ of the weight-function $H\left(\eta\left(x^{(k)}\right)\right)$ in the form:

$$
\eta^{(k)}=\frac{a_{1}}{b_{1}} I+L_{1} e^{(k)}+L_{2} e^{(k)^{2}}+L_{3} e^{(k)^{3}}+L_{4} e^{(k)^{4}}+\mathcal{O}\left(e^{(k)^{5}}\right),
$$

being $L_{1}=\frac{1}{b_{1}} I+a_{1} Y_{2}$ and $L_{t}=\frac{1}{b_{1}} N_{t}+\sum_{j=1}^{t}\left(a_{1} Y_{j+1}+Y_{j+1} N_{t-j}\right), t=2,3, \ldots$. This result allows us to expand $H\left(\eta\left(x^{(k)}\right)\right)$ around $c$ by Taylor series

$$
\begin{aligned}
H\left(\eta^{(k)}\right) & \approx H(c)+H_{1}\left(\eta^{(k)}-c\right)+\frac{1}{2} H_{2}\left(\eta^{(k)}-c\right)^{2} \\
& =H(c)+S_{1} e^{(k)}+S_{2} e^{(k)^{2}}+S_{3} e^{(k)^{3}}+S_{4} e^{(k)^{4}}
\end{aligned}
$$

where $S_{1}=H_{1} L_{1}, S_{2}=H_{1} L_{2}+\frac{1}{2} H_{2} L_{1}^{2}, S_{3}=H_{1} L_{3}+\frac{1}{2} H_{2}\left(L_{1} L_{2}+L_{2} L_{1}\right)$ and $S_{4}=H_{1} L_{4}+\frac{1}{2} H_{2}\left(L_{1} L_{3}+\right.$ $\left.L_{2}^{2}+L_{3} L_{1}\right)$.

Now,

$$
\left[F^{\prime}\left(x^{(k)}\right)\right]^{-1} F\left(y^{(k)}\right)=R_{2} e^{(k)^{2}}+R_{3} e^{(k)^{3}}+R_{4} e^{(k)^{4}}+\mathcal{O}\left(e^{(k)^{5}}\right),
$$

where $R_{2}=-A_{2}, R_{3}=-\left(A_{3}+X_{2} A_{2}\right)$ and $R_{4}=A_{5}-X_{2} A_{3}-X_{3} A_{2}$. Then,

$$
H\left(\eta^{(k)}\right)\left[F^{\prime}\left(x^{(k)}\right)\right]^{-1} F\left(y^{(k)}\right)=Q_{2} e^{(k)^{2}}+Q_{3} e^{(k)^{3}}+Q_{4} e^{(k)^{4}}+\mathcal{O}\left(e^{(k)^{5}}\right),
$$

where $Q_{2}=H(c) R_{2}, Q_{3}=H(c) R_{3}+S_{1} R_{2}$ and $Q_{4}=H(c) R_{4}+S_{1} R_{3}+S_{2} R_{2}$. Finally, we obtain the error equation in the form

$$
e^{(k+1)}=(I-H(c)) C_{2} e^{(k)^{2}}+K_{3} e^{(k)^{3}}+K_{4} e^{(k)^{4}}+\mathcal{O}\left(e^{(k)^{5}}\right) .
$$

If $H(c)=I$ we get order of convergence at least three and the error equation takes the form

$$
e^{(k+1)}=\left(2 b_{1}^{2} I-\left(a_{2} b_{1}-a_{1} b_{2}\right) H^{\prime}(c)\right) C_{2}^{2} e^{(k)^{3}}+K_{4}^{\prime} e^{(k)^{4}}+\mathcal{O}\left(e^{(k)^{5}}\right) .
$$

If $H^{\prime}(c)=\frac{2 b_{1}^{2}}{a_{2} b_{1}-a_{1} b_{2}} I$ we obtain

$$
e^{(k+1)}=\frac{1}{2 b_{1}^{4}}\left[\left(10 b_{1}^{4}+4 b_{1}^{3} b_{2}-H^{\prime \prime}(c)\left(a_{2}^{2} b_{1}^{2}-2 a_{1} a_{2} b_{1} b_{2}+a_{1}^{2} b_{2}^{2}\right)\right) C_{2}^{3}-2 b_{1}^{4} C_{2} C_{3}\right] e^{(k)^{4}}+\mathcal{O}\left(e^{(k)^{5}}\right),
$$

and the proof is finished.

Next, by choosing different weight functions and values of the parameters, we present some particular iterative schemes. We assume that

$$
H\left(\eta^{(k)}\right)=I+\frac{2 b_{1}^{2}}{a_{2} b_{1}-a_{1} b_{2}}\left(\eta^{(k)}-\frac{a_{1}}{b_{1}} I\right)
$$

where

$$
\eta^{(k)}=M^{-1} N=\left[\left(b_{1}+b_{2}\right) I-b_{2}\left[F^{\prime}\left(x^{(k)}\right)\right]^{-1}\left[x^{(k)}, y^{(k)} ; F\right]\right]^{-1}\left[\left(a_{1}+a_{2}\right) I-a_{2}\left[F^{\prime}\left(x^{(k)}\right)\right]^{-1}\left[x^{(k)}, y^{(k)} ; F\right]\right] \text {. }
$$

In this case, we note that if $b_{2}=0$ the method of Sharma et al. [6] is obtained with independence of the values of parameters $a_{1}, a_{2}$ and $b_{1}$. The next particular iterative schemes will be used in the numerical section. 
- If $a_{1}=1, a_{2}=0, b_{1}=1$ and $b_{2}=2$, we obtain the following iterative method of order four, denoted by MS1,

$$
x^{(k+1)}=y^{(k)}-\left(2 I-\left[3 I-2\left[F^{\prime}\left(x^{(k)}\right)\right]^{-1}\left[x^{(k)}, y^{(k)} ; F\right]\right]^{-1}\right)\left[F^{\prime}\left(x^{(k)}\right)\right]^{-1} F\left(y^{(k)}\right) .
$$

- If $a_{1}=1 / 2, a_{2}=0, b_{1}=-1 / 2$ and $b_{2}=1$, then the fourth-order method denoted by MS2 has the expression

$$
x^{(k+1)}=y^{(k)}-\left(2 I-\frac{1}{2}\left[\frac{1}{2} I-\left[F^{\prime}\left(x^{(k)}\right)\right]^{-1}\left[x^{(k)}, y^{(k)} ; F\right]\right]^{-1}\right)\left[F^{\prime}\left(x^{(k)}\right)\right]^{-1} F\left(y^{(k)}\right) .
$$

In both cases, $y^{(k)}$ denotes Newton's iteration.

\section{Numerical tests}

In the first part of this section we present the numerical results obtained by applying the proposed methods on some scalar equations. We are going to compare ME1 and ME2 with some fourth-order known methods as Kung-Traub scheme [1],

$$
\begin{aligned}
& y_{k}=x_{k}-\frac{f\left(x_{k}\right)}{f^{\prime}\left(x_{k}\right)}, \\
& x_{k+1}=y_{k}-\frac{f\left(x_{k}\right)^{2}}{\left(f\left(x_{k}\right)-f\left(y_{k}\right)\right)^{2}} \frac{f\left(y_{k}\right)}{f^{\prime}\left(x_{k}\right)},
\end{aligned}
$$

denoted by MKT; the scheme of Zhao et al. [17]

$$
\begin{aligned}
& y_{k}=x_{k}-\frac{f\left(x_{k}\right)}{f^{\prime}\left(x_{k}\right)}, \\
& x_{k+1}=y_{k}-\frac{1+2 u_{k}+u_{k}^{2}}{1-4 u_{k}^{2}} \frac{f\left(y_{k}\right)}{f^{\prime}\left(x_{k}\right)},
\end{aligned}
$$

denoted by MZ, where $u_{k}=\frac{f\left(y_{k}\right)}{f\left(x_{k}\right)}$; and the Jarratt-type method designed by Jaiswal [18],

$$
\begin{aligned}
& y_{k}=x_{k}-\frac{2}{3} \frac{f\left(x_{k}\right)}{f^{\prime}\left(x_{k}\right)} \\
& x_{k+1}=x_{k}-\left[2-\frac{7 f^{\prime}\left(y_{k}\right)}{4 f^{\prime}\left(x_{k}\right)}+\frac{3}{4}\left(\frac{f^{\prime}\left(y_{k}\right)}{f^{\prime}\left(x_{k}\right)+f^{\prime}\left(y_{k}\right)}\right)^{2}\right] \frac{2 f\left(x_{k}\right)}{f^{\prime}\left(x_{k}\right)+f^{\prime}\left(y_{k}\right)},
\end{aligned}
$$

denoted by MJ.

In these numerical tests, variable precision arithmetic has been used, with 2000 digits of mantissa, in Matlab R2014b on the following test functions:

- $f_{1}(x)=\sin (x)-x^{2}+1, \quad \xi \approx 1.409240 . .$,

- $f_{2}(x)=\arctan (x), \quad \xi=0$,

- $f_{3}(x)=\cos (x)-x, \quad \xi \approx 0.739085 . .$, 
- $f_{4}(x)=\sqrt{x^{2}+2 x+5}-2 \sin (x)-x^{2}+3, \quad \xi \approx 2.331968 .$. ,

- $f_{5}(x)=e^{x}-4 x^{2}, \quad \xi \approx 0.714806$..,

- $f_{6}(x)=\sqrt{x^{2}+8} \sin \left(\frac{\pi}{x^{2}+1}\right)+\frac{x^{3}}{x^{4}+1}-\sqrt{6}+\frac{8}{17}, \quad \xi=-2$.

Table 1 shows the results obtained on the test functions using as stopping criterion $\left|x_{k+1}-x_{k}\right|<10^{-500}$ or $\left|f\left(x_{k+1}\right)\right|<10^{-500}$. For each function and method, we show the initial guess $x_{0}$, the error estimations in the last iteration $\left|x_{k+1}-x_{k}\right|$ and $\left|f\left(x_{k+1}\right)\right|$, the number of iterations required to satisfy the stopping criterion, the CPU-time in seconds, $e-$ time, calculated as the average of 100 consecutive runs through the cputime command, and the computational approximate order of convergence, $\rho$, which is an approximation of the theoretical order of convergence, introduced in [19] as

$$
p \approx \rho=\frac{\ln \left(\left|x_{k+1}-x_{k}\right| /\left|x_{k}-x_{k-1}\right|\right)}{\ln \left(\left|x_{k}-x_{k-1}\right| /\left|x_{k-1}-x_{k-2}\right|\right)}
$$

valid both for equations as well as for systems of equations. The numerical tests provided in Table 1 confirm the theoretical results, with small differences between the different methods.

In the context of nonlinear systems, we are going to compare our methods MS1 and MS2 with the following known schemes:

- The derivative-free method designed by Sharma et al. [20], denoted by MSS,

$$
\begin{aligned}
y^{(k)} & =x^{(k)}-\left[x^{(k)}, u^{(k)} ; F\right]^{-1} F\left(x^{(k)}\right), \\
x^{(k+1)} & =y^{(k)}-G^{(k)}\left(3 I-2 G^{(k)}\right)\left[x^{(k)}, u^{(k)} ; F\right]^{-1} F\left(y^{(k)}\right),
\end{aligned}
$$

where $G^{(k)}=\left[x^{(k)}, u^{(k)} ; F\right]^{-1}\left[y^{(k)}, z^{(k)} ; F\right], z^{(k)}=y^{(k)}+F\left(y^{(k)}\right)$ and $u^{(k)}=x^{(k)}+F\left(x^{(k)}\right)$.

- The Jarratt-type method obtained by Hueso et al. in [21] and denoted by MSH

$$
\begin{aligned}
y^{(k)=} & x^{(k)}-\frac{2}{3}\left[F^{\prime}\left(x^{(k)}\right)\right]^{-1} F\left(x^{(k)}\right), \\
x^{(k+1)=} & x^{(k)}-\left(-\frac{3}{8} I+\left[F^{\prime}\left(y^{(k)}\right)\right]^{-1} F^{\prime}\left(x^{(k)}\right)+\frac{1}{3}\left[F^{\prime}\left(x^{(k)}\right)\right]^{-1} F^{\prime}\left(y^{(k)}\right)+\right. \\
& \left.\frac{1}{24}\left(\left[F^{\prime}\left(y^{(k)}\right)\right]^{-1} F^{\prime}\left(x^{(k)}\right)\right)^{2}\right)\left[F^{\prime}\left(x^{(k)}\right)\right]^{-1} F\left(x^{(k)}\right) .
\end{aligned}
$$

We check the performance of these methods on the following functions:

- $F_{1}\left(x_{1}, x_{2}\right)=\left(\exp \left(x_{1}\right) \exp \left(x_{2}\right)+x_{1} \cos \left(x_{2}\right), x_{1}+x_{2}-1\right)^{T}$, $\xi \approx(3.470631 \ldots,-2.470631 \ldots)^{T}$.

- $F_{2}\left(x_{1}, x_{2}, x_{3}\right)=\left(10 x_{1}+\sin \left(x_{1}+x_{2}\right)-1,8 x_{2}-\cos ^{2}\left(x_{3}-x_{2}\right)-1,12 x_{3}+\sin x_{3}-1\right)^{T}$, $\xi \approx(0.068978 \ldots, 0.246442 \ldots, 0.076929 \ldots)^{T}$.

- $F_{3}\left(x_{1}, x_{2}\right)=\left(x_{1}+\exp \left(x_{2}\right)-\cos x_{2}, 3 x_{1}-x_{2}-\sin x_{2}\right)^{T}$, $\xi=(0,0)^{T}$. 


$$
\begin{gathered}
\text { - } F_{4}\left(x_{1}, x_{2}, x_{3}\right)=\left(\cos x_{2}-\sin x_{1}, x_{3}^{x_{1}}-\frac{1}{x_{2}}, \exp \left(x_{1}\right)-x_{3}^{2}\right)^{T}, \\
\xi \approx(0.909569 \ldots, 0.661227 \ldots, 1.575834 \ldots)^{T} \\
\text { - } F_{5}\left(x_{1}, x_{2}, x_{3}, x_{4}\right)=\left(f_{1}\left(x_{1}, x_{2}, x_{3}, x_{4}\right), f_{2}\left(x_{1}, x_{2}, x_{3}, x_{4}\right), f_{3}\left(x_{1}, x_{2}, x_{3}, x_{4}\right), f_{4}\left(x_{1}, x_{2}, x_{3}, x_{4}\right)\right)^{T}, \text { where } \\
f_{1}\left(x_{1}, x_{2}, x_{3}, x_{4}\right)=x_{2} x_{3}+x_{4}\left(x_{1}+x_{3}\right), \\
f_{2}\left(x_{1}, x_{2}, x_{3}, x_{4}\right)=x_{1} x_{3}+x_{4}\left(x_{1}+x_{3}\right), \\
f_{3}\left(x_{1}, x_{2}, x_{3}, x_{4}\right)=x_{1} x_{2}+x_{4}\left(x_{1}+x_{2}\right), \\
f_{4}\left(x_{1}, x_{2}, x_{3}, x_{4}\right)=x_{1} x_{2}+x_{1} x_{3}+x_{2} x_{3}-1, \\
\xi \approx(0.577350 \ldots, 0.577350 \ldots, 0.577350 \ldots,-0.288675 \ldots)^{T},
\end{gathered}
$$

by using a symmetric divided difference operator.

The numerical results shown in Table 2, have been obtained with MatlabR2014b working in variable precision arithmetics with 50 digits of mantissa and by using the stopping criterion $\left\|x^{(k+1)}-x^{(k)}\right\|<10^{-25}$ or $\left\|F\left(x^{(k+1)}\right)\right\|<10^{-25}$. For each test function and method we show the error estimations in the last iteration $\left\|x^{(k+1)}-x^{(k)}\right\|$ and $\left\|F\left(x^{(k+1)}\right)\right\|$, the number of iterations required to satisfy the stopping criterion and the computational approximate order of convergence, $\rho$, calculated by (9). Symbol '-' denotes that the corresponding method does not converge with the initial guess used.

The worse behavior of method MSS reflected in Table 2 (examples $F_{1}, F_{2}$ and $F_{4}$ ) is due to the unstable performance of methods that only use divided differences. However, methods that uses a combination of Jacobian matrices and divided differences, or only Jacobian matrices, are more stable and their numerical tests confirm the theoretical results.

\section{Conclusions}

We have designed an optimal (in the sense of Kung-Traub conjecture) family of scalar methods constructed from Traub's scheme and by using the weight functions technique. We have applied a technique for generalizing this family to nonlinear systems, preserving the order of convergence. Although this technique has been applied to two-step iterative methods, it could be applied to many multipoint iterative schemes that there exist in the literature. Numerical results show that some of the designed iterative methods for nonlinear systems are very competitive in relation to other well known ones.

Acknowledgments: The authors thank to the anonymous referees for the valuable comments and suggestions that have improved the final version of the paper.

[1] H.T. Kung, J.F. Traub, Optimal order of one-point and multipoint iteration, J. Assoc. Comput. Math. 21 (1974) 643-651.

[2] M.F. Abad, A. Cordero, J.R. Torregrosa, A family of seventh-order schemes for solving nonlinear systems, Bull. Math. Soc. Sci. Math. Roumanie 57(105) No. 2 (2014) 133-145.

[3] A. Cordero, J.G. Maimó, J.R. Torregrosa, M.P. Vassileva, Solving nonlinear problems by Ostrowski-Chun type parametric families, J. Math. Chem. 52 (2014) 430-449.

[4] S. Artidiello, Diseño, implementación y convergencia de métodos iterativos para resolver ecuaciones y sistemas no lineales utilizando funciones peso, Ph.D. Thesis, Servicio de publicaciones Universidad Politécnica de Valencia, 2014, https://riunet.upv.es/handle/10251/321. 
[5] J. R. Sharma, R. K. Guna, R. Sharma, An efficient fourth order weighted-Newton method for systems of nonlinear equations, Numer. Algor. 62 (2013) 307-323.

[6] J.R. Sharma, H. Arora, On efficient weighted-Newton methods for solving systems of nonlinear equations. Appl. Math. Comput. 222 (2013) 497-506.

[7] C. Andreu, N. Cambil, A. Cordero, J.R. Torregrosa, Preliminary Orbit Determination of Artificial Satellites: A Vectorial Sixth-Order Approach, Abstr. Appl. Anal., 2013 (Article ID 960582).

[8] M. F. Abad, A. Cordero, J. R. Torregrosa, Fourth-and fifth-order for solving nonlinear systems of equations: An application to the global positioning system, Abstr. Appl. Anal., 2013 (Article ID 586708).

[9] M. Petković, B. Neta, L. Petković, J. Džunić, Multipoint Methods for Solving Nonlinear Equations, Academic Press, Amsterdam, 2013.

[10] J.F. Traub, Iterative Methods for the Solution of Equations, second ed., Prentice Hall, New York, 1964.

[11] S. Artidiello, F. Chicharro, A. Cordero and J.R. Torregrosa, Local convergence and dynamical analysis of a new family of optimal fourth-order iterative methods, Inter. J. of Comp. Math. 90(10) (2013) 2049-2060.

[12] C. Chun, Some fourth-order iterative methods for solving nonlinear equations, Appl. Math. Comput. 195 (2008) 454-459.

[13] R.F. King, A family of fourth order methods for nonlinear equations, SIAM J. Numer. Anal. 10 (1973) 876-879.

[14] J.M. Ortega, W.C. Rheinboldt, Iterative Solutions of Nonlinears Equations in Several Variables, Academic Press, New York, 1970.

[15] C. Hermite, Sur la formule d'interpolation de Lagrange, J. Reine Angew. Math. 84 (1878) 70-79.

[16] A. Cordero, J.L. Hueso, E. Martínez, J.R. Torregrosa, A modified Newton-Jarratt's composition, Numer. Algor. 55 (2010) 87-99.

[17] L. Zhao, X. Wan g, W. Guo, New families of eighth-order methods with high efficiency index for solving nonlinear equations, Wseas Transac. Math. 11 (2012) 283-293.

[18] J.P. Jaiswal, Some class of thrid- and fourth-order iterative methods for solving nonlinear equations, J. Appl. Math., 2014 (Article ID 817656).

[19] A. Cordero, J.R. Torregrosa, Variants of Newton's method using fifth-order quadrature formulas, Appl. Math. Comput. 190 (2007) 686-698.

[20] J.R. Sharma, H. Arora, M. Petković, An efficient derivative free family of fourth order methods for solving systems of nonlinear equations, Appl. Math. Comput. 235 (2014) 383-393.

[21] J.L. Hueso, E. Martínez, C. Teruel, Convergence, efficiency and dynamics of new fourth and sixth order families of iterative methods for nonlinear systems, J. Comput. Appl. Math. 275 (2015) 412-420. 


\begin{tabular}{|c|c|c|c|c|c|c|}
\hline & Method & $\left|x_{k+1}-x_{k}\right|$ & $\left|f\left(x_{k+1}\right)\right|$ & iter & $\rho$ & $e-$ time \\
\hline \hline$f_{1}(x)=0$ & ME1 & $2.9 \mathrm{e}-251$ & $1.3 \mathrm{e}-1002$ & 6 & 4.0000 & 0.0978 \\
$x_{0}=1$ & ME2 & $2.0 \mathrm{e}-315$ & $2.1 \mathrm{e}-1259$ & 6 & 4.0000 & 0.0864 \\
& MKT & $6.5 \mathrm{e}-421$ & $1.6 \mathrm{e}-1681$ & 6 & 4.0000 & 0.0917 \\
& MZ & $1.3 \mathrm{e}-249$ & $4.7 \mathrm{e}-998$ & 6 & 4.0000 & 0.1100 \\
& MJ & $2.9 \mathrm{e}-271$ & $1.3 \mathrm{e}-1082$ & 6 & 4.0000 & 0.0964 \\
\hline \hline$f_{2}(x)=0$ & ME1 & $1.3 \mathrm{e}-225$ & $7.8 \mathrm{e}-1126$ & 6 & 5.0000 & 0.0852 \\
$x_{0}=1$ & ME2 & $2.6 \mathrm{e}-371$ & $2.8 \mathrm{e}-1854$ & 6 & 5.0000 & 0.0741 \\
& MKT & $1.0 \mathrm{e}-110$ & $2.1 \mathrm{e}-546$ & 5 & 5.0000 & 0.0657 \\
& MZ & $1.4 \mathrm{e}-160$ & $1.4 \mathrm{e}-800$ & 6 & 5.0000 & 0.0803 \\
& MJ & $1.1 \mathrm{e}-235$ & $2.1 \mathrm{e}-1176$ & 6 & 5.0000 & 0.0917 \\
\hline \hline$f_{3}(x)=0$ & ME1 & $1.1 \mathrm{e}-190$ & $1.6 \mathrm{e}-761$ & 5 & 4.0000 & 0.0685 \\
$x_{0}=1.5$ & ME2 & $8.7 \mathrm{e}-194$ & $4.6 \mathrm{e}-774$ & 5 & 4.0000 & 0.0583 \\
& MKT & $1.8 \mathrm{e}-197$ & $5.8 \mathrm{e}-789$ & 5 & 4.0000 & 0.0627 \\
& MZ & $1.5 \mathrm{e}-208$ & $1.2 \mathrm{e}-833$ & 5 & 4.0000 & 0.0772 \\
& MJ & $3.6 \mathrm{e}-195$ & $1.5 \mathrm{e}-779$ & 5 & 4.0000 & 0.0817 \\
\hline \hline$f_{4}(x)=0$ & ME1 & $2.6 \mathrm{e}-231$ & $6.0 \mathrm{e}-925$ & 5 & 4.0000 & 0.2677 \\
$x_{0}=3$ & MED2 & $8.7 \mathrm{e}-295$ & $9.4 \mathrm{e}-1179$ & 5 & 4.0000 & 0.2576 \\
& MKT & $1.4 \mathrm{e}-232$ & $7.4 \mathrm{e}-930$ & 5 & 4.0000 & 0.2624 \\
& MZ & $9.9 \mathrm{e}-197$ & $2.2 \mathrm{e}-786$ & 5 & 4.0000 & 0.2781 \\
& MJ & $2.6 \mathrm{e}-240$ & $3.6 \mathrm{e}-961$ & 5 & 4.0000 & 0.2819 \\
\hline \hline$f_{5}(x)=0$ & ME1 & $1.1 \mathrm{e}-254$ & $1.2 \mathrm{e}-1015$ & 6 & 4.0000 & 0.0970 \\
$x_{0}=2$ & ME2 & $1.4 \mathrm{e}-268$ & $2.2 \mathrm{e}-1071$ & 6 & 4.0000 & 0.0855 \\
& MKT & $1.1 \mathrm{e}-286$ & $6.1 \mathrm{e}-1144$ & 6 & 4.0000 & 0.0909 \\
& MZ & $2.5 \mathrm{e}-369$ & $1.0 \mathrm{e}-1475$ & 6 & 4.0000 & 0.1090 \\
& MJ & $5.4 \mathrm{e}-264$ & $6.2 \mathrm{e}-1053$ & 6 & 4.0000 & 0.1145 \\
\hline \hline$f_{6}(x)=0$ & ME1 & $7.1 \mathrm{e}-135$ & $3.6 \mathrm{e}-536$ & 5 & 4.0000 & 0.3824 \\
$x_{0}=-1$ & ME2 & $1.7 \mathrm{e}-142$ & $8.7 \mathrm{e}-567$ & 5 & 4.0000 & 0.3831 \\
& MKT & $5.9 \mathrm{e}-153$ & $8.1 \mathrm{e}-609$ & 5 & 4.0000 & 0.3763 \\
& MZ & $9.2 \mathrm{e}-201$ & $4.1 \mathrm{e}-801$ & 5 & 4.0000 & 0.3927 \\
& MJ & $7.2 \mathrm{e}-138$ & $3.3 \mathrm{e}-548$ & 5 & 4.0000 & 0.3962 \\
\hline \hline
\end{tabular}

Table 1: Numerical results for nonlinear equations 


\begin{tabular}{|c|c|c|c|c|c|}
\hline & Method & $\left\|x^{(k+1)}-x^{(k)}\right\|$ & $\left\|F\left(x^{(k+1)}\right)\right\|$ & iter & $\rho$ \\
\hline \hline$F_{1}$ & MS1 & $2.1 \mathrm{e}-16$ & $3.4 \mathrm{e}-49$ & 3 & 3.483 \\
$x^{(0)}=(3,-2)^{T}$ & MS2 & $7.9 \mathrm{e}-17$ & $7.6 \mathrm{e}-50$ & 3 & 3.601 \\
& MSS & - & - & - & - \\
& MSH & $5.7 \mathrm{e}-16$ & $1.3 \mathrm{e}-57$ & 3 & 3.608 \\
\hline \hline$F_{2}$ & MS1 & $5.1 \mathrm{e}-12$ & $6.2 \mathrm{e}-40$ & 3 & 4.455 \\
$x^{(0)}=(1,1,1)^{T}$ & MS2 & $3.2 \mathrm{e}-14$ & $1.2 \mathrm{e}-44$ & 3 & 4.093 \\
& MSS & $2.9 \mathrm{e}-8$ & $2.9 \mathrm{e}-29$ & 3 & 2.383 \\
& MSH & $9.0 \mathrm{e}-18$ & $1.6 \mathrm{e}-58$ & 3 & 4.115 \\
\hline \hline \multirow{2}{*}{$F_{3}^{(0)}=(1,1)^{T}$} & MS1 & $2.7 \mathrm{e}-15$ & $1.4 \mathrm{e}-44$ & 4 & 3.889 \\
& MS2 & $2.8 \mathrm{e}-7$ & $1.7 \mathrm{e}-27$ & 3 & 3.380 \\
& MSS & $8.0 \mathrm{e}-25$ & $5.3 \mathrm{e}-50$ & 5 & 4.011 \\
& MSH & $4.2 \mathrm{e}-22$ & $2.0 \mathrm{e}-79$ & 4 & 3.963 \\
\hline \hline \multirow{2}{*}{$x_{4}^{(0)}=(1,1,2)^{T}$} & MS1 & $3.4 \mathrm{e}-17$ & $7.8 \mathrm{e}-49$ & 5 & 3.762 \\
& MS2 & $1.7 \mathrm{e}-9$ & $4.13 \mathrm{e}-33$ & 3 & 3.766 \\
& MSS & - & - & - & - \\
& MSH & $2.4 \mathrm{e}-25$ & $6.4 \mathrm{e}-58$ & 4 & 3.918 \\
\hline \hline \multirow{2}{*}{$F_{5}^{(0)}=(1,1,1,1)^{T}$} & MS1 & $2.9 \mathrm{e}-22$ & $3.5 \mathrm{e}-58$ & 4 & 4.448 \\
& MS2 & $3.4 \mathrm{e}-8$ & $1.4 \mathrm{e}-32$ & 3 & 4.557 \\
& MSS & $1.1 \mathrm{e}-24$ & $4.2 \mathrm{e}-58$ & 6 & 4.025 \\
& MSH & $4.7 \mathrm{e}-7$ & $1.0 \mathrm{e}-29$ & 3 & 4.471 \\
\hline \hline
\end{tabular}

Table 2: Numerical results for nonlinear systems 